\title{
On the Evaluation of Determinants, the Formation of their Adjugates, and the Practical Solution of Simultaneous Linear Equations
}

By A. C. Aitken, Edinburgh University.

(Received 20th October, 1932. Read 3rd December, 1932.)

\section{INTRODUCTORY.}

There are various methods in existence for the practical solution of a set of simultaneous equations,

$$
a_{i 1} x_{1}+a_{i 2} x_{2}+\ldots+a_{i n} x_{n}=c_{i}, \quad i=1,2, \ldots, n .
$$

Some of these methods are appropriate to special systems, as for example to the axisymmetric "normal equations" of Least Squares. In many applications, however, as in problems of statistical correlation of many variables, it may be desired not merely to solve a given set of equations but to obtain as much knowledge as possible about the system or matrix of coefficients; perhaps to evaluate its determinant and various minors, such as principal minors, possibly also to determine the elements of the adjugate matrix, or the reciprocal matrix. The examination of the sign of successive principal minors of an axisymmetric determinant, in order to find the signature of the corresponding quadratic form, is a case in point; and there are many such applications.

It seems to us, after considerable numerical practice, that if we are granted the use of a machine capable of forming and adding or subtracting binary products, both positive and negative, and of dividing the resulting sums by arbitrary divisors, then the elementary and old-established process of reduced elimination can be so arranged as to provide almost everything that is desired. The eliminations, which in practice are simply cross-multiplications supplemented by divisions at the proper stage, lead to successively condensed systems, the elements of which are signed minors of the original system, the 
sign being determined most easily by the use of Sylvester's " umbral notation "1 and the usual rule of inversion of indices. By augmenting the original array with suitable columns containing zeros and units, in fact with a "unit matrix," the scheme can be used at the same time to form the adjugate matrix (by solving the set of equations, in matrix or vector notation, $A x=y$ ), and therefore, if desired, the reciprocal matrix; and an additional column can be kept for a "check sum." The method could likewise be used to solve the more general system of equations of transformation,

$$
a_{i 1} x_{1}+a_{i 2} x_{2}+\ldots+a_{i n} x_{n=}=b_{i 1} y_{1}+b_{i 2} y_{2}+\ldots+b_{i n} y_{n},
$$

in matrix notation $A x=B y$, and hence to evaluate the matrix $A^{-1} B$.

Methods of the kind described below have been used for two or three years by the author and by students working under him, and have been found well adapted to machine work. The present paper, which has been in manuscript a considerable time, is now published in the hope that, while of little theoretical importance, it may be of interest and value to computers.

At the suggestion of an interested friend the author some time ago applied the routine to the set of six normal equations by which Gauss in 1809 calculated $^{2}$ the corrections to the elements of the minor planet Pallas. This particular set of equations has a formidable appearance, owing to the wide range of magnitude of the numbers involved; but the solution was performed, and the results verified by substitution in all six equations-a check well worth applying in any circumstances-in some two hours or less. The results proved to be in considerable disagreement with those of Gauss. Our conjecture is that Gauss misread the coefficient [ab], or 7203.91, as $7039 \cdot 21$, an error (arising from transposition of figures) which threw out the calculations almost at the very beginning. At this late date

1 According to which the minor, e.g. arising from the 1st, 3rd and 4th rows, and 2nd, 5th and 6th columns, is represented by $\left(\begin{array}{lll}1 & 3 & 4 \\ 2 & 5 & 6\end{array}\right)$.

2 Werke VI, 18-24; see also Bertrand's translation in his Méthode des Moindres Carrés, 139-141. 
the circumstance has not much interest, and certainly no importance; and we mention it solely to account for the discrepancy.

In an appendix we describe by means of a brief example a method put forward by T. Smith 1 for building up the adjugate in stages, a method which does not seem to be widely known, and which, subject to the reservations we mention, might often be employed with advantage.

\section{Arrangement of Reduced Elimination.}

The process of reduced elimination consists in the repeated use of such determinantal identities as

$$
\begin{aligned}
& || a_{1} b_{2} c_{3} d_{4}|| b_{2} c_{3} d_{4} e_{5}||=\left|a_{1} b_{2} c_{3} d_{4} e_{5}\right| \cdot\left|b_{2} c_{3} d_{4}\right|, \\
& \left|a_{1} b_{2} c_{3} d_{4} e_{5}\right|=|| a_{1} b_{2} c_{3} d_{4}|| b_{2} c_{3} d_{4} e_{5}|| \div\left|b_{2} c_{3} d_{4}\right|
\end{aligned}
$$

where determinants, and compound determinants, such as that on the left of (1), are represented by their diagonal elements. The identity ( 1 ), in Muir's terminology, is simply the "extensional," by means of the extension ${ }^{2}\left[b_{2} c_{3} d_{4}\right]$, of the trivial identity

$$
|| a_{1}|| e_{5}||=\left|a_{1} e_{5}\right| \text {. }
$$

By way of illustration of the method we shall first set out the solution of a set of equations and the calculation of the elements of the adjugate matrix of the system, choosing a rather simple example and including a check sum. The first equation of the system is

$$
17 x_{1}-9 x_{2}+12 x_{3}-22 x_{4}=-3
$$

which will show how the others are entered in the scheme. The rows and columns in successive stages on the left are named "umbrally" by bracketed indices depending on the position of the "pivotal elements" used in preceding stages. The use of these will be described in more detail in a moment. The work is set out thus:

' Phil. Mag. (3), 18 (1927), 1007.

2 An extension, i.e. added letters and suffixes, can of course be inserted not merely at the end, as $\left|a_{1} b_{2}\right|$ becoming $\left|a_{1} b_{2} c_{3} d_{4}\right|$, but in the middle or elsewhere, as $\left|a_{1} d_{4}\right|$ becoming $\left|a_{1} b_{2} c_{3} d_{4}\right|$. 


$$
\begin{aligned}
& \begin{array}{lll} 
& x \\
\text { (1) } & \text { (2) (3) (4) }
\end{array} \\
& \text { (1) } \quad \begin{array}{llll}
17 & -9 & 12 & -22
\end{array} \\
& \text { I. (2) } \quad 19 \quad-11 \quad 15 \quad-27
\end{aligned}
$$

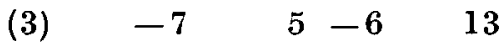

$$
\begin{aligned}
& \text { (4) } \quad 31 \quad-18 \quad 24 \quad-37 \\
& \begin{array}{llll}
\text { (13) } & 22 & 6 & 6
\end{array} \\
& \text { II. } \begin{array}{rrrrr}
(23) & 18 & . & 9 & \frac{8}{79} \\
(43) & 29 & . & 12 & 49
\end{array}
\end{aligned}
$$

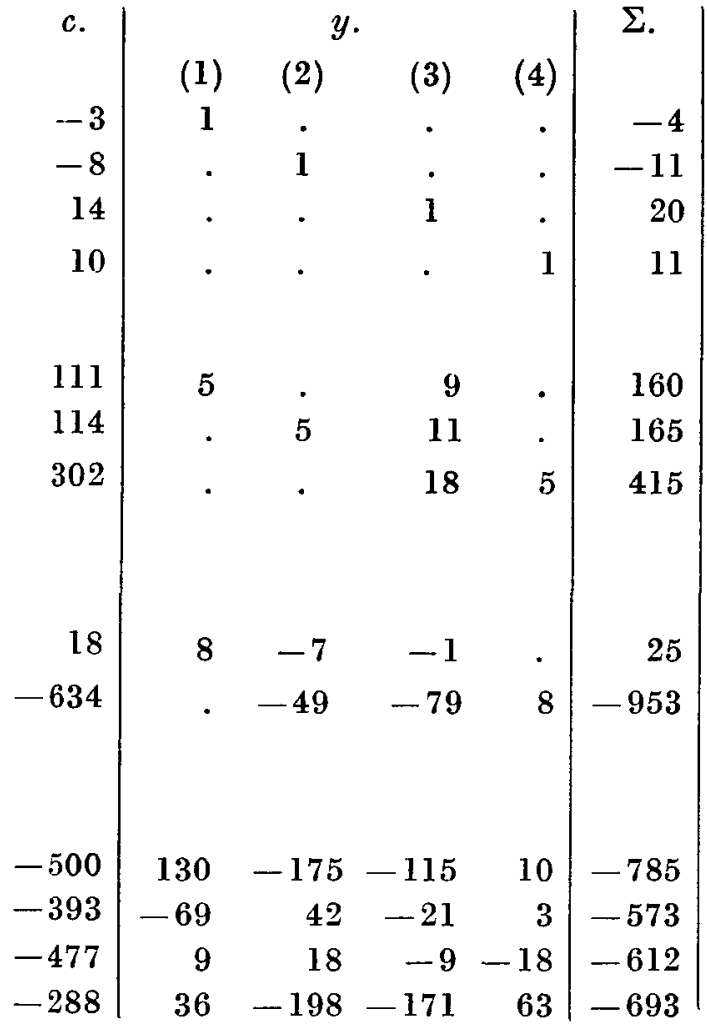

$$
\begin{aligned}
& \text { (4321) . . }-135 \text {. } \\
& \begin{array}{ccccc}
\div 10 . & -135 & . & . & . \\
\div 8 & . & . & . & -135
\end{array} \\
& \div 5 \text {. } \quad-135
\end{aligned}
$$

Explanation. The arrangement at Stage I hardly calls for description. The last column contains the check sums, that is, the sums of all the entries in the respective rows. A pivotal element is chosen at will, as here the underlined 5, and elimination is performed with respect to the variable it affects. The procedure is simply to evaluate, in the whole array $I$ of four rows and ten columns, every minor of the second order which involves this 5, treating the diagonal which includes the 5 as the principal diagonal however it is actually placed; that is, giving its own sign to the cross-product involving the 5, and reversing the sign of the other cross-product. For example, the entry 22 at Stage II comes from (5) (17) - (-7) (-9), the entry 29 from $(5)(31)-(-7)(-18)$, and the entry 415 in the check column from (5) (11) - (20) (-18). It is easily proved that the check value, as computed in this way by cross multiplication, ought always to agree with the sum of the elements in the row in question, and the verification should be made as each row is completed. 
At Stage II we choose another pivot at will, namely the underlined 8 , and cross-multiply with respect to it in the same way, dividing each result, however, by the previous pivot, 5. For example the entry -69 at Stage III comes from (8)(12) - (9) (49), divided by 5 . It is a consequence of the identity (1) that such division, when the cross-multiplications have been extended to their full accuracy, should be exact without remainder. In ordinary numerical practice, however, it would be uselessly laborious to retain all the digits necessary for such a check, unless indeed all the elements were small; and the check sum is used instead.

Later stages $^{1}$ follow the same rule, that is, cross-multiplication about a chosen pivot, supplemented by division by the previous pivot. For example the ultimate single entry -135 at Stage IV comes from $(10)(-69)-(-3)(-130)$, divided by 8 . (It will be explained later why this result is entered in four places in Stage IV.) This actually gives with correct sign the value of the determinant $\Delta$ of the system, but it is necessary now to describe by what rule of sign we are able to assert this, as well as to fix the sign of the various signed minors which appear as entries in the intermediate stages.

Rule of Sign. Row indices are put at the left side of the rows and column indices at the heads of columns. The first index within a bracket names the row or column of the original array from which the entry in question has been derived. When at any stage we use a pivot named by brackets with first row index $i$, first column index $j$, we annex $i$ and $j$ respectively as last indices in the umbral brackets which designate the rows and columns of the next stage. Inspection will show that this has been done in our example. It may then be proved without trouble ${ }^{2}$ by aid of the identity (1) that all entries are thus designated umbrally in the notation of Sylvester with correct sign. (For example the entry -3 at Stage III would be written as $\left(\begin{array}{l}132 \\ 324\end{array}\right)$, namely the minor based on rows $1,3,2$, columns $3,2,4$ of the

1 If the original determinant is of less than full rank, we shall come to a stage in which all entries are zero. Of. I. H. Rice, Journ. Math. and Phys. (Massachusetts), 11 (1932), 146-149.

${ }^{2} C f$. $\$ 5$, infra. This rule of sign differs from that given by the author in a very similar method for computing determinants, Trans. Fac. Act., 13 (1931), 272-275. 
original array, taken in that order.) To fix the sign the ordinary rule of inversions of indices may be used. The writer here often adopts the simple expedient of writing the row indices above the column indices, joining by a line each row index to the same column index, and giving plus or minus as the number of intersections of the lines is even or odd.

We could of course avoid all trouble regarding sign by choosing always either the leading element or indeed any diagonal element of an array for pivot, as one would naturally do in solving a symmetric system of normal equations, - for then the minors would all be entered with their own sign; but in a non-symmetric system it might well happen that diagonal elements were either zero or else so small as to have large tabular uncertainty, in which case the choice of a nondiagonal element for pivot would be enforced.

At row (4321) of Stage IV the original equations have been reduced to "triangular form." To complete the solution we might copy out, for example, equations (4321), (132), (13) and (1) from the scheme, and solve in succession. It is neater and easier, however, to choose the solving set according to the pivots already used, and to use the latter once more in reversed order, proceeding thus:

Enter the value of $\Delta$ in a new row under the last pivot, 10, as shown. Cross-multiply in row (132), which contains the pivot 10, with respect to the underlined -135 or $\Delta$ in the row (4321), dividing all results by the pivot 10 . For example the entry -393 comes from $(-135)(18)-(-500)(-3)$ divided by 10 , and so on.

Next, enter again a $\Delta$, or -135 , in a new row under the still earlier pivot 8, and perform cross multiplications with respect to the two earlier $\Delta$ 's together, using row (23), in which the 8 occurs, and dividing by the 8 . For example the entry -477 comes from $(-135)(114)-(-500)(9)-(-393)(18)$, divided by 8 ; the entry -612 from $(-135)(165)-(-785)(9)-(-573)(18)$, divided by 8 .

Last of all, enter another $\Delta$, or -135 , under the first pivot used, 5, and compute, e.g. -288 as $(-135)(14)-(-500)(-6)-(-393)(-7)$ $-(-477)(13)$, divided by 5 .

This step completes the work. Rearranging the rows in Stage IV so that the $\Delta$ 's fall in the diagonal, we write down the solution of the proposed equations as

$$
x_{1}=\frac{393}{135}=2 \cdot 911, x_{2}=\frac{288}{135}=2 \cdot 133, x_{3}=\frac{500}{135}=3 \cdot 704, x_{4}=\frac{477}{135}=3 \cdot 533 .
$$


At the same time the adjugate matrix, by the same rearrangement, is found from the rows on the right of Stage IV to be

$$
\operatorname{adj} A=\left|\begin{array}{rrrr}
-69 & 42 & -21 & 3 \\
36 & -198 & -171 & 63 \\
130 & -175 & -115 & 10 \\
9 & 18 & -9 & -18
\end{array}\right|
$$

We use the word adjugate strictly in the matrix sense, which involves co-factors or signed minors in the elements, and differs from the determinantal definition, as ordinarily given, in that rows replace columns and vice versa. By dividing each element of the adjugate by $\Delta$ we obtain the reciprocal matrix.

It is believed that, with experience and practice, the above method will be found uniform and straightforward.

\section{Example of a Set of Normal Equations.}

In the present section we shall set out the solution of the normal equations in the astronomical problem of Gauss, earlier mentioned. The coefficients themselves are in some cases in slight disagreement with those given by Gauss; this is due to the fact that on discovering the discrepancy in the solution, and conjecturing the reason, we went through for ourselves the work of deriving the six normal from the eleven observational equations, and found a few disagreements too large to be explained by the ordinary rounding off of results to such and such a number of significant figures. We also solved the normal equations using Gauss's values (after replacing 7032.91 by 7203.91), obtaining results which hardly differed from those obtained below.

The computation has been restricted to five significant digits, and a characteristic index in heavy type has been used to indicate the order of magnitude. E.g. 3.12345 would represent $123 \cdot 45$, and $\overline{3} \cdot 12345$ would represent 0.00012345 .

In each stage the leading element is pivot, a choice which makes the method here resemble Gauss's own method, the point of difference being that we perform the divisions a stage later than he did, thereby obtaining minors, and finally the determinant, of the system. In symmetric systems the choice of a diagonal pivot preserves symmetry and thus practically halves the labour of computation. 
$x$.

$y . \quad z$.

$u$.

$v$.

$w$.

$c$.

$\Sigma$.

(1) 1.59157

$4.72039-\overline{1} \cdot 93458-1 \cdot 22851-0.34664-0.18197$

$3 \cdot 37109$

$4 \cdot 75780$

(2) 4.72039

$8 \cdot 10834-2 \cdot 49064-4 \cdot 32298-3 \cdot 19864-3 \cdot 14306$

$6 \cdot 58010$

$8 \cdot 11418$

(3) $-\overline{\mathbf{1}} \cdot 93458$

$\begin{array}{llll}0.71919 & 1.11338 & \overline{1} .58616 & 0.26284\end{array}$

$3 \cdot 11334$

$2 \cdot 66357$

(4) $-1 \cdot 22851-4 \cdot 32298$

$1 \cdot 11338$

$2 \cdot 12003-0.37137-0.12040$

$-3 \cdot 26839$

$-4 \cdot 34878$

(5) $-0.34664-3 \cdot 19864$

$\overline{1} .58616-0.37137 \quad 1.22821-0.36261$

$-2 \cdot 94274$

$-3 \cdot 29165$

(6) $-0.18197-3 \cdot 14306$

$0.26284-0.12040-0.36261 \quad 1.56246$

$2 \cdot 31764$

$-3 \cdot 10607$

$\begin{array}{rr}(12) & 8 \cdot 12195 \\ (13) & 3 \cdot 38301 \\ (14) & -4 \cdot 26449 \\ (15) & 4 \cdot 13221 \\ (16) & 3 \cdot 46459\end{array}$

$3 \cdot 38301-4.26449 \quad 4 \cdot 13221 \quad 3.46459$

$6 \cdot 75840$

$8 \cdot 12953$

$1 \cdot 42458$

$1 \cdot 64937$

$0 \cdot 31436 \quad 1 \cdot 15379$

$3 \cdot 70517$

$4 \cdot 11008$

$1 \cdot 64937$

$2 \cdot 65784-1 \cdot 29890-1 \cdot 11281$

$-3 \cdot 73974$

$-4 \cdot 33165$

$\begin{array}{llllll}0.31436 & -1 \cdot 29890 & 2 \cdot 13380 & -1 \cdot 22082 & -3.42906\end{array}$

$3 \cdot 90154$

$1 \cdot 15379-1 \cdot 11281-1 \cdot 22082 \quad 2 \cdot 33240$

$3 \cdot 25543$

$3 \cdot 75146$ $\div 1 \cdot 59157$

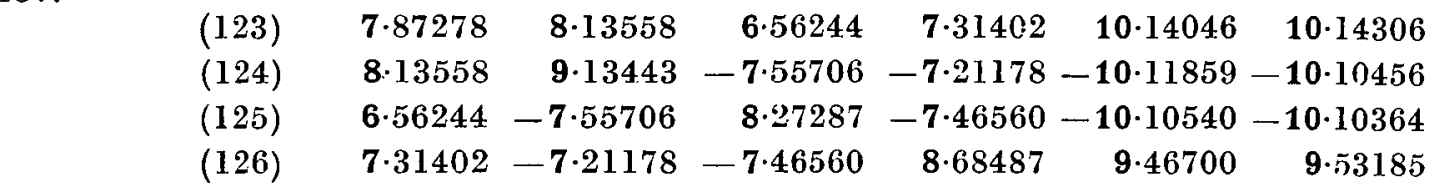
$\div 8 \cdot 12195$.

$\div \mathbf{7} \cdot 87278$

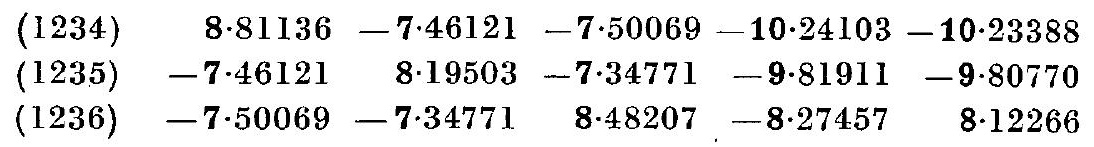

$$
\begin{array}{rrr}
(12345) & 9 \cdot 17887 & -8 \cdot 34970-10 \cdot 88884-10 \cdot 87445 \\
(12346) & -8 \cdot 34970 & 9 \cdot 44527-10 \cdot 16380-10 \cdot 12277
\end{array}
$$

\section{$\div 8 \cdot 81136$}

$\begin{array}{cccccccc}-2.15593 & \mathbf{1} .53996 & 3.21841 & -\mathbf{2 . 3 3 0 9 2} & -2.51197 & -\mathbf{1 . 7 6 9 9 5} & \mathbf{1} \cdot 1 & -\mathbf{1 . 6 6 9 9 5} \\ (1) & (2) & (3) & (4) & (5) & (6) & \\ 3.37109 & 6.58010 & 3.11334 & -3.26839 & -2.94274 & 2.31762 \text { (check by substitution) }\end{array}$

Thus $x=-15.593 ; y=0.053996 ; z=218.41 ; u=-33.092 ; v=-51.197 ; w=-7.6995$.

The values given by Gauss (loc. cit.) were

$x=-3 \cdot 06, y=0 \cdot 054335, z=166 \cdot 44, u=-4 \cdot 29, v=-34 \cdot 37, w=-3 \cdot 15$.

It may be verified that these values do not satisfy the original equations. They do not even satisfy equation (1), which was used by Gauss in finding the last of his unknowns; but it may be noted that they would do so, if it were again allowed that 7203.91 had been misread or miscopied as $7032 \cdot 91$.

In conclusion we may make some general remarks. Suppose that, in some non-symmetric system, we were working to four significant 
digits, and had a choice between pivotal elements $0 \cdot 8723$ and $0 \cdot 1078$. It would be natural in most cases to choose the former, since its tabular uncertainty is only about one eighth of that of the other, and this will be of importance when it comes to be used as a divisor. On the other hand it does not follow that such a choice will be vindicated at the next stage, for the minors resulting from the chosen pivot may have much greater tabular uncertainty than those resulting from the one rejected. The truth is that, whereas in the ordinary algebraic theory of matrices and determinants with exact elements many questions (e.g. questions of rank) hinge simply on whether minors are exactly zero or not zero, in the statistical and observational use of these functions we have ${ }^{1}$ varying degrees of sensitiveness to error in the minors, and the fixing of a limit to the error committed by the result may indeed be a very difficult matter. There can be no doubt whatever that in common practice considerations like these are either lost sight of or treated perfunctorily.

4. Appendix on T. Smith's Method of Forming the Adjugate.

For the theory of T. Smith's method of building up the adjugate in stages we refer to his paper itself, cited earlier, remarking only that his rule for computing interior elements, within the border, can be looked upon, and might have been demonstrated, as a direct consequence of Jacobi's theorem on any minor of the adjugate. We shall display the practical working of the method by using it to calculate the value of the numerical determinant used in our own example of $\S 2$, and the elements of its adjugate. The work appears thus:

I.

1

\begin{tabular}{|c|c|c|c|c|c|c|c|c|}
\hline \multirow{2}{*}{ II. } & -19 & 12 & & \multirow{3}{*}{-16} & \multirow{2}{*}{\multicolumn{4}{|c|}{ A. }} \\
\hline & 17 & 15 & & & & & & \\
\hline-7 & 5 & -6 & & & 17 & -9 & 12 & -22 \\
\hline-9 & 9 & 18 & -22 & \multirow{4}{*}{-18} & 19 & -11 & 15 & -27 \\
\hline 6 & -18 & -22 & -27 & & -7 & 5 & -6 & .13 \\
\hline 111. -3 & -27 & -16 & 13 & & 31 & -18 & 24 & -37 \\
\hline 31 & -18 & 24 & -37 & & & & & \\
\hline \multirow{3}{*}{ IV. } & 36 & 130 & 9 & \multirow{4}{*}{-135} & & & & \\
\hline & -198 & -175 & 18 & & & & & \\
\hline & -171 & -115 & -9 & & & & & \\
\hline 3 & 63 & 10 & -18 & & & & & \\
\hline
\end{tabular}

I Gf. I. M. H. Etherington, in Proc. Edin. Math. Soc. (2), 3 (1932), 107-117. 
Explanation. Stage I simply contains the leading element of the system. At Stage II the adjugate (in the sense used in the theory of determinants, without transposition of rows and columns) of the leading minor of the second order is written down, as shown within the dotted lines, and the numerical value of the minor is entered in the column headed by $\Delta_{r}$. The adjugate is then bordered by elements from the third row and column of the original determinant.

At Stage III we use these bordering elements as multipliers thus: the entry -3 comes from $(12)(-11)+(15)(9)$ with sign altered; the entry -27 from $(12)(-19)+(15)(17)$, with sign altered. Similarly the entry 18 comes from $(-7)(-11)+(5)(-19)$, with sign altered; and the entry -22 below it from $(-7)(9)+(5)(17)$, with sign altered. The corner element -16 is just the value of $\Delta_{r}$ at the previous stage.

The determinant of the partial adjugate at Stage III is now easily calculated, for the entries $-3,-27,-16$ are the co-factors of the entries $-7,5,-6$ in the border of Stage II, so that we now enter $(-7)(-3)+(5)(-27)+(-6)(-16)$, or -18 , in the $\Delta$ column; a result which is checked at once by $(12)(18)+(15)(-22)+(-6)(-16)$. The interior elements at Stage III are now calculated thus: e.g. the entry -9 comes from $(-18)(-11)+(18)(-3)$, divided by -16 , where the -11 occupies the same place in Stage II that this -9 is going to occupy in Stage III, the -18 and -16 are the respective $\Delta$ 's, and the 18 and -3 are the already calculated exterior elements in the same row and column as the -9. Each interior element is calculated in a similar manner. The whole is then bordered with elements from the fourth row and column of the original determinant, and first the border, then the interior elements of the final adjugate at Stage IV are derived in the same manner as at Stage III.

Thus the determinantal adjugate is derived. The more useful matrix adjugate is obtained by transposition of rows and columns.

. It will be seen that the arrangement is ingenious and effective. It must however be pointed out that there are situations which would throw it into difficulties. One is the case in which any intermediate $\Delta_{r}$ proved to be either zero, in which case it could not be used as a divisor at the next stage to find the interior elements, or else small and with large tabular uncertainty. The former disability would not arise with a set of normal equations, for the matrix of such a set is necessarily positive definite, so that the principal minors $\Delta_{r}$ are essentially positive and non-zero; but they might quite conceivably 
be small. There is a similar caveat regarding Dodgson's method of evaluating determinants; and one may say indeed that any system of solving equations or of computing determinants which does not possess flexibility of choice in divisors is likely to be thrown out by circumstances of the kind we have mentioned.

\section{A Note on Extensional Identities. (Added Nov. 12, 1932.)}

The rule of sign of the working process of $\S 2$ may perhaps be elucidated by the following brief account of those types of determinantal identity which Muir has named "complementary" and "extensional" respectively.

Jacobi's theorem ${ }^{1}$ on a minor of the adjugate states that any minor of the adjugate, e.g. $\left|A_{1} B_{2} C_{3} D_{4}\right|$ of a determinant $\Delta=\left|a_{1} b_{2} c_{3} d_{4}\right|$ (determinants being indicated here by their diagonal elements), is equal to the complementary minor of the determinant mulliplied by a suitable power of $\Delta$. For example,

$$
\left|B_{2} C_{3}\right|=\left|a_{1} d_{4}\right| \Delta, \quad\left|A_{1} C_{3} D_{4}\right|=b_{2} \Delta^{2} \text {, and so on. }
$$

Here let us observe that $\Delta$ itself may conveniently be considered as a minor, its complement being unity, which we may regard as a minor of zero order.

It follows at once from Jacobi's theorem that to every homogeneous identity involving minors taken from a general array, such as $\left(a_{1} b_{2} c_{3} d_{4}\right)$, there corresponds a complementary identity, obtained by writing complementary capital letters and suffixes instead of the original small letters and suffixes. Further, the elements of the original array being quite general, so also are those of the adjugate array; whence we infer that the complementary identities are not merely valid qua complementary, but are valid in their own right, so that one may quite well replace capital letters by small.

We thus arrive at the true conception of the duality of complementary identities, while we also observe the reflexive property, by which the complementary of a complementary identity, with respect to the original array, restores the original identity. But here one might proceed otherwise; from a complementary one might proceed to form a second complementary, not with respect to the original array but with respect to an augmented or extended array, such as $\left(a_{1} b_{2} c_{3} d_{4} e_{5} f_{6}\right)$. In such a case we return, not to the original identity,

${ }^{3}$ Cf. Turnbull, Determinants, Matrices and Invariants, 77-79. 
but to one derived from it by elongating each diagonal representation of a minor by the "extension," e.g. $\left(e_{5} f_{6}\right)$, and in most cases, for purposes of homogeneity, inserting powers of the determinant $\left|e_{5} f_{6}\right|$, which may be regarded as the extension of a minor of zero order. These new identities are what Muir has called "extensionals" of the original ones; and the discrimination of such types effectively condenses much of the older literature of determinants.

To take a simple example, so trivial an identity as

$$
|| a_{1}|| b_{2}||=\left|a_{1} b_{2}\right|
$$

yields, by an extension like $\left(c_{3} d_{4}\right)$, the by no means obvious identity in compound determinants

$$
|| a_{1} c_{3} d_{4}|| b_{2} c_{3} d_{4}||=\left|a_{1} b_{2} c_{3} d_{4}\right| \cdot\left|c_{3} d_{4}\right| .
$$

Writing the last identity umbrally as

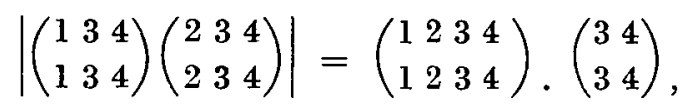

we observe that extensionals can be written in umbral notation with equal vividness, and in some respects with more flexibility, since upper and lower indices may be freely permuted. The extension here is $\left(\begin{array}{ll}3 & 4 \\ 3 & 4\end{array}\right)$.

The rule of sign of $\S 2$ will now be seen at once to be based on umbral extensionals. For example, if we agree to write the pivotal element last in diagonal representations, we may observe that the passage from Stage III to Stage IV in the example of $\S 2$ is based on the extensional

$$
\left|\left(\begin{array}{lll}
4 & 3 & 2 \\
3 & 2 & 4
\end{array}\right)\left(\begin{array}{lll}
1 & 3 & 2 \\
1 & 2 & 4
\end{array}\right)\right|=\left(\begin{array}{llll}
4 & 3 & 2 & 1 \\
3 & 2 & 4 & 1
\end{array}\right) \cdot\left(\begin{array}{ll}
3 & 2 \\
2 & 4
\end{array}\right),
$$

where $\left(\begin{array}{ll}3 & 2 \\ 2 & 4\end{array}\right)$, that is $\left(\begin{array}{ll}2 & 3 \\ 4 & 2\end{array}\right)$, is the pirotal element at the previous Stage II. The effect has been to attach first upper and lower indices of the pivot at III as last indices of the entry at IV, and the reader can easily build an induction to show that in the arrangement adopted this must always hold.

As a final example of the use of extensionals, we shall derive the important expansion of Schweins $^{1}$ for the quotient of two

1 Muir, History of Determinants, Vol I, Part I, p. 172. 
determinants differing in one column (or row) only. Thus for the fourth order the theorem is

$$
Q_{4}=\frac{\left|\theta_{1} b_{2} c_{3} d_{4}\right|}{\mid a_{1} b_{2} c_{3} d_{4}}=\frac{\theta_{1}}{a_{1}}+\frac{\left|\theta_{1} a_{2}\right| b_{1}}{a_{1}\left|a_{1} b_{2}\right|}+\frac{\left|\theta_{1} a_{2} b_{3}\right|\left|b_{1} c_{2}\right|}{\left|a_{1} b_{2}\right|\left|a_{1} b_{2} c_{3}\right|}+\frac{\left|\theta_{1} a_{2} b_{3} c_{4}\right|\left|b_{1} c_{2} d_{3}\right|}{\left|a_{1} b_{2} c_{3}\right|\left|a_{1} b_{2} c_{3} d_{4}\right|} \text {. }
$$

To prove this, we have, if $Q_{3}$ is the quotient for the third order,

(1) $Q_{4}-Q_{3}=\| \theta_{1} b_{2} c_{3} d_{4}|| a_{1} b_{2} c_{3}|| \div\left|a_{1} b_{2} c_{3}\right| \cdot\left|a_{1} b_{2} c_{3} d_{4}\right|$.

The numerator is the extensional, by $\left(b_{2} c_{3}\right)$, of $\left\|\theta_{1} d_{4}|| a_{1}\right\|$, that is, of $\left|\theta_{1} a_{4}\right| . d_{1}$. Hence its value is $\left|\theta_{1} b_{2} c_{3} a_{4}\right| \cdot\left|d_{1} b_{2} c_{3}\right|$, or, by compensating interchanges of columns, $\left|\theta_{1} a_{2} b_{3} c_{4}\right| \cdot\left|b_{1} c_{2} d_{3}\right|$.

Now substituting in (1), and adding the analogous results for $Q_{3}-Q_{2}, Q_{2}-Q_{1}, Q_{1}$, we derive the Schweins expansion. 